\title{
Comparison of the effect of rapeseed oil or amaranth seed oil supplementation on weight loss, body composition, and changes in the metabolic profile of obese patients following 3-week body mass reduction program: a randomized clinical trial
}

Małgorzata Moszak ${ }^{1 *}$ D, Agnieszka Zawada², Aldona Juchacz ${ }^{3}$, Marian Grzymisławski ${ }^{2}$ and Paweł Bogdański ${ }^{1}$

\begin{abstract}
Background: Amaranth seed oil (ASO) and rapeseed oil (RSO) are functional foods that display antioxidant and hepatoprotective properties. These oils are also known to lower glucose and cholesterol levels. The current study compared the effects exerted by RSO and ASO on weight loss and metabolic parameters during a 3-week body mass reduction program.

Methods: Eighty-one obese subjects $\left(\mathrm{BMl}>30 \mathrm{~kg} / \mathrm{m}^{2}\right)$, aged 25-70 years, were enrolled in a 3-week body mass reduction program based on a calorie-restricted diet and physical activity. Participants were randomly categorized into an AO group (administered $20 \mathrm{~mL} / \mathrm{d}$ of ASO), a RO group (administered $20 \mathrm{~mL} / \mathrm{d}$ of RSO), and a C group (control; untreated). Anthropometric and metabolic parameters were measured at baseline and endpoint.

Results: Significant decreases in weight, body mass index (BMI), waist circumference (WC), hip circumference (HC), fat mass (FM), lean body mass (LBM), visceral fat mass (VFM), and total body water (TBW\%) were observed in all groups $(P<0.05)$. No significant improvements were observed in the clinical parameters of group C. Fasting insulin $(\Delta-5.9$, and $\Delta-5.7)$ and homeostatic model assessment of insulin resistance (HOMA-IR) $(\Delta-1.1$ and $\Delta-0.5)$ were decreased in both $\mathrm{RO}$ and $\mathrm{AO}$ groups, respectively. Fasting glucose $(\Delta-8.5 ; P=0.034)$, total cholesterol $(\Delta-14.6 ; P=0.032)$, non$\mathrm{HDL}$ cholesterol $(\Delta 15.9 ; P=0.010), \mathrm{TG} / \mathrm{HDL}$ ratio $(\Delta-0.6 ; P=0.032)$, LDL cholesterol $(\Delta-12.3 ; P=0.042)$, and triglycerides $(\Delta-6.5 ; P=0.000)$ were significantly improved in the $\mathrm{AO}$ group, compared to the $\mathrm{RO}$ group.

\footnotetext{
* Correspondence: mmoszak@ump.edu.pl

'Department of Obesity and Metabolic Disorders Treatment and Clinical

Dietetics, Karol Marcinkowski University of Medical Sciences, ul.

Szamarzewskiego 82/84, 60-569 Poznań, Polska, Poland

Full list of author information is available at the end of the article
}

C C The Author(s). 2020 Open Access This article is licensed under a Creative Commons Attribution 4.0 International License, which permits use, sharing, adaptation, distribution and reproduction in any medium or format, as long as you give appropriate credit to the original author(s) and the source, provide a link to the Creative Commons licence, and indicate if changes were made. The images or other third party material in this article are included in the article's Creative Commons licence, unless indicated otherwise in a credit line to the material. If material is not included in the article's Creative Commons licence and your intended use is not permitted by statutory regulation or exceeds the permitted use, you will need to obtain permission directly from the copyright holder. To view a copy of this licence, visit http://creativecommons.org/licenses/by/4.0/ The Creative Commons Public Domain Dedication waiver (http://creativecommons.org/publicdomain/zero/1.0/) applies to the data made available in this article, unless otherwise stated in a credit line to the data. 


\begin{abstract}
(Continued from previous page)
Conclusions: The 3-week body mass reduction intervention caused a significant reduction in the weight, BMI, WC, HC, FM, and VFM of all groups. Except for HOMA-IR, there were no statistical differences between the clinical parameters of all groups. However, a trend toward improved insulin levels and $\mathrm{HDL} \%$ was noticeable in $\mathrm{AO}$ and RO. Therapies involving edible oils with high nutritional value, such as RSO and ASO, show potential for improving metabolic measurements during body mass reduction programs. Thus, obese patients undertaking weight reduction programs may benefit from RSO and ASO supplementation.
\end{abstract}

Trial registration: retrospectively registered, DRKS00017708.

Keywords: Amaranth seed oil, Body composition, Metabolic profile, Rapeseed oil, Weight loss

\section{Background}

Obesity, which is a significant public health issue, has reached pandemic levels in the developed world [1]. According to 2016 World Health Organization (WHO) statistics, 39\% of the population was overweight and 650 million (13\%) were obese [2]. Lifestyle changes which focus on proper nutrition and physical activity constitute the primary approach for treating obesity [3]. Functional food consumption has been suggested as another useful method for reducing the prevalence of obesity. Functional foods are defined as foods fortified with usually scarce nutrients or foods without harmful ingredients (e.g. allergens), which provide health benefits. For example, Cicero et al. [4] described lipid- and blood pressure-lowering properties of bioactives such as berberine, plant sterols, green tea extract, soy, curcumin, cocoa, pycnogenol, lycopene, olive oil, soluble fibers, garlic, resveratrol, beetroot, and mineral salts. Although functional food products deliver additional or enhanced benefits over and above their basic nutritional value, these foods should not be considered as alternatives to a balanced diet $[5,6]$.

Amaranth seed oil (ASO) and rapeseed oil (RSO) are functional food products that are becoming increasingly popular. The health-promoting properties of both oils are mainly due to a high content of monounsaturated fatty acids (MUFAs) and polyunsaturated fatty acids (PUFAs). In addition, properties of both oils are determined by a unique composition of bioactive substances as follows: squalene, sterols, tocopherols, carotenoids, phospholipids, etc., for ASO; and tocopherols, carotenoids, flavonoids, phytosterols, and phenolic links (sinapine) for RSO [7, 8]. However, ASO and RSO differ in popularity, availability and price. RSO is one of the most popular and commonly used oils in Poland, while ASO is considered to be "exotic" and "healthier" than RSO [9].

Previous studies have indicated that diets supplemented with ASO improved antioxidant properties, and exhibited anti-inflammatory, hypotensive, and hepatoprotective effects [10]. Furthermore, Kim et al. [11] investigated the effect of amaranth seed and amaranth oil supplementation on the blood glucose profiles of streptozotocin-induced diabetic rats. The hypoglycemic activity of ASO has not yet been established via clinical trials. RSO, which also possesses similar antioxidant, hypolipemic, antiinflammatory and anti-atherogenic properties, is cheaper and more ubiquitous than ASO $[12,13]$. Previous studies have shown that modulation of a fatty acid profile by converting fats commonly present in diet, such as RSO, has a positive effect on carbohydrate metabolism and reduces the risk of developing breast cancer [7].

However, studies comparing the effectiveness of ASO and RSO on weight reduction and normalization of metabolic parameters associated with obesity are lacking. Fatty acid compositions of ASO and RSO are different. RSO contains higher levels of MUFAs and PUFAs than ASO and displays a more beneficial PUFA/saturated fatty acid (SFA) ratio and unsaturated fatty acid (USFA)/ SFA ratio than those of ASO. However, ASO is rich in squalene $[14,15]$. The fatty acid composition of both oils is shown in Table 1.

The objective of this study was to compare the effect of ASO and RSO supplementation on anthropometric parameters (body mass, body mass index (BMI), waist circumference (WC), hip circumference (HC), waist/hip ratio (WHR), and body composition) and selected biochemical parameters (fasting serum glucose and insulin levels, homeostatic model assessment of IR (HOMA-IR), total cholesterol (TC), high-density lipoprotein (HDL), low-density lipoprotein (LDL), triglycerides (TG), nonHDL levels and TG/HDL ratios in obese adults following a strictly controlled weight loss program lasting 3-weeks.

\section{Methods \\ Research design}

The study was designed as a randomized, double-blind, controlled trial with 3 parallel groups, and performed at the Department of Gastroenterology, Internal Diseases and Dietetics, from July 2014 to March 2016. The study protocol was approved by the Research Ethics Committee of the Poznan University of Medical Sciences, Poland (approval 333/14), and the study was performed in accordance with the Helsinki Declaration. All patients provided signed informed consent prior to participation in 
Table 1 Fatty acid composition of ASO and RSO

\begin{tabular}{lll}
\hline Component & Amaranth seed oil & Rapeseed oil \\
\hline 16:0 (palmitic acid) SFA & $18.5-23.4 \%$ & $2.9 \%$ \\
$16: 1$ (palmitoleic acid) & $0.09-0.4 \%$ & $0.2 \%$ \\
18:0 (stearic acid) SFA & $3.4-4.5 \%$ & $4.5 \%$ \\
$18: 1$ n-9 (oleic acid) MUFA & $22.6-26.0 \%$ & $59 \%$ \\
18:2 n-6 (linoleic acid) PUFA omega 6 & $38.2-49.9 \%$ & $21 \%$ \\
$18: 3$ n-3 (a-linolenic) PUFA omega 3 & $0.92-1.2 \%$ & $11.2 \%$ \\
$20: 1$ n-9 (eicosenoic acid) & 0.1 & $1.4 \%$ \\
$22: 0$ (behenic acid) & $0.1-0.4 \%$ & $2 \%$ \\
$22: 1$ (euric acid) & & $0.1 \%$ \\
$24: 0$ (lignoceric acid) & $0.1-0.4 \%$ & $1.5 \%$ \\
Squalene & $58.8-77.7 \mathrm{mg} / \mathrm{g}$ & $<0.05 \mathrm{mg} / \mathrm{g}$ \\
Phytosterols & $1931-2762 \mathrm{mg} / 100 \mathrm{~g}$ & $558-1407 \mathrm{mg} / 100 \mathrm{~g}$ \\
Tocopherols & $656.8-2588 \mathrm{mg} / \mathrm{kg}$ & $620-950 \mathrm{mg} / \mathrm{kg}$ \\
\hline
\end{tabular}

the study. The study was retrospectively registered in the Deutsches Register Klinischer Studien under the number DRKS00017708.

\section{Participants}

One hundred and six obese Polish adults were enrolled. Inclusion criteria for the study were $B M I \geq 30 \mathrm{~kg} / \mathrm{m}^{2}$, stable body weight $(<3 \mathrm{~kg}$ self-reported change during the previous 3 months) and being 18-70 years old. The exclusion criteria were as follows: (i) secondary form of obesity; (ii) chronic diseases related to metabolism (e.g., chronic liver, kidney, pancreas diseases, inborn metabolic diseases, autoimmune diseases, inflammatory bowel diseases, coeliac disease); (iii) diabetes mellitus type 1 or uncontrolled diabetes mellitus type 2; (iv) uncontrolled disorders of lipid metabolism; (v) vegetarian or any another alternative dietary habit; (vi) a history of using any dietary supplements within the 3 months prior to the study; (vii) diagnosed with hyperlipidemia or hypertension, or diabetes mellitus type 2 , or requiring the introduction and/or change of pharmacological treatment for these diseases during the 2 years before the trial; (viii) eating disorders; and (ix) nicotine, alcohol or drug abuse. Prior to randomization, 9 subjects were excluded according to exclusion criteria, while 2 withdrew consent. The remaining 95 patients were randomized and subjected to intervention. During the course of the 3-week period, 14 patients failed to complete the study as follows: 4 from C, 5 from AO, and 5 from RO. Finally, data pertaining to 81 participants were analyzed. Among those analyzed, 49 had hypertension, 27 had type 2 diabetes and 35 had hyperlipidemia. However, these subjects maintained a constant pattern of pharmacotherapy in accordance with exclusion criteria. The flowchart of the study is shown (Fig. 1).

\section{Interventions}

Each eligible and consenting participant was assigned a unique code as an identifier. The study population was randomly assigned to 3 treatment groups as follows: 20 $\mathrm{mL}$ ASO (AO group; 16 women, 10 men); $20 \mathrm{~mL}$ RSO (RO group, 16 women, 10 men); and untreated (C group (control); 19 women, 10 men). Participants and investigators were blinded to randomization and group allocation. Patients from all groups underwent a 3-week body mass reduction program conducted under controlled conditions. The duration of the study was determined to be 3-weeks due to the need to hospitalize participants for the entire intervention period. Thus, the body mass reduction program was implemented during a 21-day hospital stay. The study participants did not have an opportunity to meet people other than medical staff or obtain food except for the meals provided by a specialized food caterer.

During the 3-week body mass reduction program, each subject received daily physical training, in the form of aerobics, from a physical therapist as well as a hypocaloric diet $(70-75 \%$ of the total daily energy expenditure (TDEE)). The TDEE was individually tailored for each participant using the Harris-Benedict formula and the physical activity level (PAL) index. In order to determine the TDEE and assess the real energy value of the diet, before the program, subjects were required to complete a 24-h record for 3 days. All subjects received the same type of diet prepared by a dietetic food caterer based on a planned menu. Each patient received a diet with an identical composition of macronutrients (20\% protein, 25-30\% fat, and 50-55\% carbohydrates) derived from the same products. The diets were supplemented with $20 \mathrm{~mL}$ ASO (AO group) or $20 \mathrm{~mL}$ RSO (RO group) per day instead of the $20 \mathrm{~g}$ of fat (butter) normally included 
Assessed for eligibility
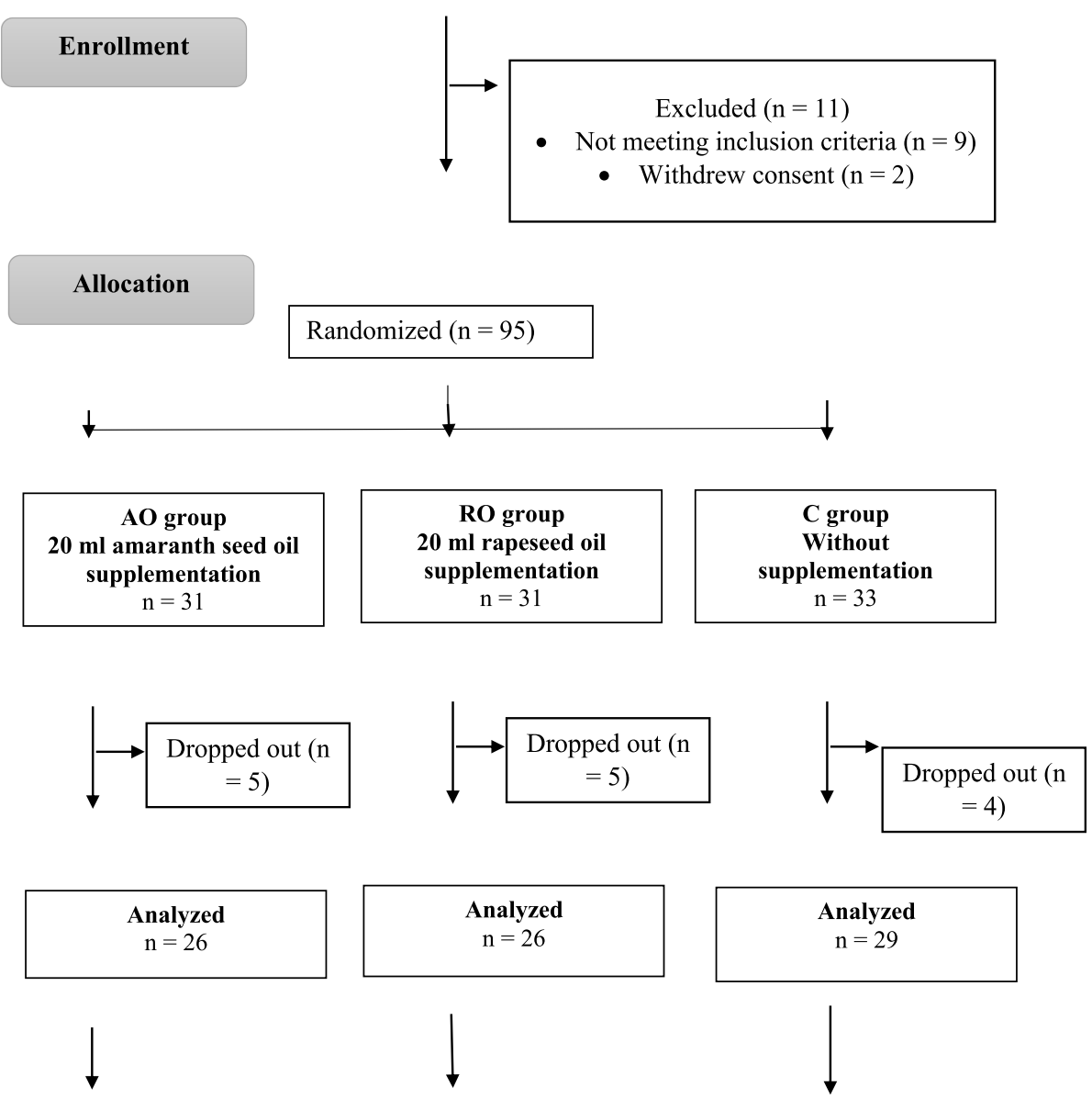

\section{Analysis}

Fig. 1 Study flowchart

in the diet. One meal (breakfast) was supplemented with one dose of oil. ASO "Ol'Amar" produced by "Szarłat" (Lomza, Poland; cold-pressed amaranth oil containing squalene (5.0-6.5 g/100 mg) and vitamin E (5-10 mg/ $100 \mathrm{~g}$ ) and RSO produced by "Ol'Vita (Marcinowice, Poland; cold-pressed rapeseed oil containing omega 9 (60\%), vitamin E $(66.4 \mathrm{mg} / 100 \mathrm{~g})$ at a $\mathrm{n}-6: \mathrm{n}-3$ ratio of 2.5:1) were used. Each dose of the oil supplement and the meal was delivered to the participant by a nurse. Compliance with the study protocol was assessed daily by a nurse and weekly by a dietician.

Physical training included active and passive breathing exercises for $30 \mathrm{~min}$ a day, cardiovascular aerobic exercises twice daily for $60 \mathrm{~min}$ and resistance training for $30 \mathrm{~min}$ daily. The aerobic training session consisted of a $5 \mathrm{~min}$ warm-up session (stretching exercises) at low intensity, followed by 45 min of training (Nordic walking or cycling) at an intensity between 50 and $70 \%$ of the maximum heart rate, and $5 \mathrm{~min}$ of closing stretching and breathing exercises of low intensity. Resistance training consisted of a 5 min warm-up session (stretching exercises) of low intensity, followed by $20 \mathrm{~min}$ of exercises with a neck barbell. Kettlebells and gymnastic ball and a 5 min warm-up session (stretching exercises) at low intensity.

\section{Anthropometric assessment}

Anthropometric and metabolic parameters were assessed at baseline and endpoint for each patient. WC $(\mathrm{cm})$ was measured at the midpoint between the lower margin of the least palpable rib and top of the iliac crest, while $\mathrm{HC}$ was measured around the widest portion of the buttocks with the tape held parallel to the floor. Both measurements were 
performed using stretch-resistant medical tape (Seco) and repeated twice to obtain a certain result. Height was measured to the nearest centimeter using a stadiometer. Weight and body composition were assessed using bioelectrical impedance, Tanita MC 980 MA (Tanita, Tokyo, Japan). Body composition analysis was performed in accordance with the recommendations of the European Society for Clinical Nutrition and Metabolism (ESPEN) [16]. BMI $\left(\mathrm{kg} / \mathrm{m}^{2}\right)$ was defined as the individual's body mass divided by the square of their height. WHR was estimated as the ratio of WC to the $\mathrm{HC}$.

\section{Clinical assessment}

Biochemical and anthropometric measurements were made at the baseline and endpoint. Fasting venous blood samples $(10 \mathrm{~mL})$ were collected at the beginning and the end of the program. Venous blood samples $(10 \mathrm{~mL})$ were collected by qualified nurses. Collection was performed between 8:00 and 10:00 am under conditions of fasting after overnight resting tubes containing biological material were coded according to the system used. In order to obtain serum, blood was centrifuged at $1500 \times g$ for 10 min and preserved in $2 \mathrm{~mL}$ Eppendorf tubes. Serum glucose, TC, HDL, and TG levels were analyzed using a fully automated Modular P-800 Roche (Diamond Diagnostics, Budapest, Hungary). LDL was indirectly measured using the Friedewald equation as follows: LDL $(\mathrm{mg} / \mathrm{dL})=\mathrm{TC}-\mathrm{HDL}-(\mathrm{TG} / 5)$ [17]. Fasting insulin levels were determined via a microparticle enzyme immunoassay (Abbot, Abbot Park, IL, USA). The sensitivity of the assay, as reported by the manufacturer, was represented by a mean minimum detectable value of $1.0 \mu \mathrm{U} /$ $\mathrm{mL}$. HOMA-IR was calculated using the following formula: fasting insulin $(\mu \mathrm{U} / \mathrm{L}) \times$ fasting glucose $(\mathrm{nmol} / \mathrm{L}) /$ 22.5 [18].

\section{Statistical analysis}

Statistical analyses were performed using Statistica 10.0 (StatSoft) and the MetaboAnalyst 3.0. server (www.metaboanalyst.ca). Normality of data was checked using the Shapiro-Wilks test. Normally distributed data are expressed as mean $\pm \mathrm{SD}$, while skewed data are represented by the median (interquartile range). Differences among the 3 groups were determined using the Kruskal-Wallis test followed by an appropriate post-hoc test (multiple comparison test) or one-way ANOVA followed by Tukey's post-hoc test for normally distributed data. In order to test the differences between the baseline and endpoint in each group, the Wilcoxon test or paired $t$ test (for normally distributed data) was conducted [19]. Statistical significance was set at $P<0.05$ [20].

\section{Results}

Eighty-one obese patients (mean BMI: $39.6 \pm 7.4 \mathrm{~kg} / \mathrm{m}^{2}$ ) were enrolled in the study. Baseline anthropometric and biochemical characteristics of $\mathrm{AO}, \mathrm{RO}$ and $\mathrm{C}$ groups are shown (Tables 2 and 3). The differences between baseline metabolic and anthropometric variables of the 3 groups were not significant.

Statistically significant $(P<0.05)$ decreases in body weight, BMI, WC, HC, FM, LBM, VFM, and TBW\% were observed in all study groups following the 3-week body mass reduction program. There were significant differences between the changes caused by intervention in WC $(P=0.022), \mathrm{HC}(P=0.007)$ and VFM $(P=0.020)$ of the $\mathrm{RO}, \mathrm{AO}$, and $\mathrm{C}$ groups. (Table 4). The most significant reduction in WC and $\mathrm{HC}$ were observed in the AO group, while the most marked change in VFM was observed in $\mathrm{C}$ group.

At the end of study, no significant improvements were observed in the clinical parameters of group $\mathrm{C}$, whereas reductions in fasting insulin and HOMA-IR were observed in $\mathrm{RO}$ and $\mathrm{AO}$ groups. However, a significant reduction in fasting glucose levels was noted only in the AO group. Similarly, improvements in lipid parameters (TC, non-HDL, LDL, and TG) were also observed only in the AO group. An increase in HDL\% was seen in both $\mathrm{AO}$ and $\mathrm{RO}$ groups. Furthermore, the results revealed significant differences in HOMA-IR between RO, AO and $\mathrm{C}$ groups (Table 5).

\section{Discussion}

This is the first study which directly compared the effects of RSO or ASO supplementation on weight loss, as well as anthropometric and metabolic parameters, of obese participants of a weight loss program. RSO and ASO are characterized by a sizeable difference in fatty acid composition. ASO contains lower amounts of MUFAs ( 24\% vs. $\sim 59 \%)$ and LC n-3 PUFAs ( 1\% vs. $\sim 11 \%)$ and displays poorer PUFA/SFA and USFA/SFA ratios compared with those of RSO. Furthermore, ASO has a lower n-3/n-6 ratio than that of RSO [21]. By contrast, ASO is an oil with one of the highest squalene content. Strong anticancer, antioxidant, drug carrier, detoxifier, skin hydrating and emollient activities of squalene have been reported in both animal models and in vitro environments [22].

In contrast to studies based on long-term body mass reduction protocols, the current study evaluated whether a short-term weight loss program, conducted under strictly controlled conditions, would induce satisfactory anthropometric and metabolic changes in adult patients with obesity.

A previous study showed that the replacement of usual edible oils with oils rich in USFAs, such as ASO and RSO, resulted in lipid-modulating, anti-atherogenic, antioxidative, anti-inflammatory, hepatoprotective and 
Table 2 Baseline anthropometric characteristics of $\mathrm{RO}, \mathrm{AO}$, and $\mathrm{C}$ groups

\begin{tabular}{lllll}
\hline Variable & RO group $(\boldsymbol{n}=26)$ & AO group $(\boldsymbol{n}=26)$ & $\mathrm{C}(\boldsymbol{n}=29)$ & $\boldsymbol{P}$-value \\
\hline Weight, kg & $120.5(102.9-135)$ & $118.5(108.0-132.4)$ & $115.0(101.4-133.3)$ & $0.823^{\mathrm{b}}$ \\
BMI, kg/m & $39.8(36.9-44.0)$ & $40.6(36.7-44.3)$ & $38.4(36.1-41.7)$ & $0.490^{\mathrm{b}}$ \\
WC, cm & $126.3 \pm 13.3$ & $124.3 \pm 17.2$ & $122.3 \pm 20.6$ & $0.600^{\mathrm{a}}$ \\
HC, cm & $128.0 \pm 13.3$ & $130.6 \pm 12.9$ & $1.02 \pm 0.20 .6$ & $0.113^{\mathrm{a}}$ \\
WHR & $0.99 \pm 0.09$ & $0.95 \pm 0.1$ & $40.9 \pm 6.7$ & $0.112^{\mathrm{b}}$ \\
FM, \% & $41.0 \pm 5.4$ & $42.1 \pm 6.1$ & $46.7(40.4-54.1)$ & $0.641^{\mathrm{a}}$ \\
FM, kg & $46.9(41.9-56.2)$ & $49.7(41.0-59.6)$ & $70.1(58.5-80.4)$ & $0.594^{\mathrm{b}}$ \\
LBM, kg & $66.9(58.6-75.6)$ & $68.7(56.5-77.2)$ & $16.0(12.0-20.0)$ & $0.810^{\mathrm{b}}$ \\
VFM, n & $15.0(12.0-21.0)$ & $14.0(13.0-21.0)$ & $41.8(39.0-44.9)$ & $0.971^{\mathrm{b}}$ \\
TBW, \% & $41.6(40.3-44.5)$ & $41.4(38.7-44.7)$ & $48.6(41.6-57.0)$ & $0.792^{\mathrm{b}}$ \\
TBW, kg & $49.1(43.7-58.9)$ & $50.6(42.6-58.1)$ & $22.1 \pm 3.5$ & $0.783^{\mathrm{b}}$ \\
ECW, kg & $22.7 \pm 2.9$ & $23.1 \pm 4.2$ & $26.0(21.4-31.8)$ & $0.700^{\mathrm{b}}$ \\
ICW, kg & $25.6(23.0-32.9)$ & $27.9(22.5-33.5)$ & $0.800^{\mathrm{b}}$ \\
\hline Nom
\end{tabular}

Normally distributed data are represented by the mean \pm SD and skewed data by the median (interquartile range). $P$-value $<0.05$ is considered significant a one-way ANOVA, 'bruskal-Wallis test,

Abbreviations: $B M I$ body mass index, ECW extracellular water, FM fat mass, $H C$ hip circumference, ICW intracellular water, $L B M$ lean body mass, $T B W$ total body water, VFM visceral fat mass, WC waist circumference, WHR waist-to-hip ratio

hypotensive effects $[7,12]$. The properties of dietary fat reportedly modulated obesity by interacting with genes encoding fatty acid metabolism, adipogenesis and endocannabinoid system [23].

Animal studies have indicated that LC n-3 PUFAs may protect against weight gain, raising the possibility that LC n-3 PUFA facilitates weight loss or differential changes in body composition when incorporated into weight-loss programs [23]. Furthermore, Borsonelo et al. [24] demonstrated anxiolytic-like effects of PUFA enriched diets on animal anxiety models. A timedependent effect of LC n-3 PUFAs on weight loss in humans has been reported [25, 26]. Certain studies have shown that MUFAs that induce higher energy expenditure, diet-induced thermogenesis, and fat oxidation than that by PUFA diets, may affect weight loss more effectively than PUFAs [27, 28]. However, the current study was unable to confirm whether oil supplementation during a weight reduction program increases the effectiveness of interventions. At the end of the study, significant reductions in weight, $\mathrm{BMI}, \mathrm{WC}, \mathrm{HC}$, and FM were observed in each group, and also in subjects from the control group. Except for WC, HC and VFM, no significant differences were observed in weight loss or other anthropometric parameters between groups. Weight loss and improvement in body composition observed in this

Table 3 Baseline clinical characteristic of $\mathrm{RO}, \mathrm{AO}$, and $\mathrm{C}$ groups

\begin{tabular}{|c|c|c|c|c|}
\hline Variable & RO group $(\boldsymbol{n}=26)$ & AO group $(\boldsymbol{n}=26)$ & $C(\boldsymbol{n}=29)$ & $P$-value \\
\hline Age & $50.7 \pm 13.5$ & $46.6 \pm 10.4$ & $49.9 \pm 13.4$ & $0.273^{\mathrm{a}}$ \\
\hline Fasting plasma glucose, mg/dL & $105.0(98.0-119.0)$ & $110.5(95.0-131.0)$ & $104.0(98.0-114.0)$ & $0.852^{b}$ \\
\hline Fasting insulin, $\mu \mathrm{U} / \mathrm{L}$ & $18.5(11.3-29.3)$ & $22.6(13.2-27.5)$ & $15.5(13.0-22.2)$ & $0.591^{b}$ \\
\hline HOMA-IR & $4.8(2.9-7.5)$ & $6.1(3.2-8.9)$ & $4.0(3.3-5.3)$ & $0.410^{\mathrm{b}}$ \\
\hline $\mathrm{TC}, \mathrm{mg} / \mathrm{dL}$ & $196.6 \pm 39.5$ & $193.8 \pm 37.0$ & $192.5 \pm 47.8$ & $0.944^{\mathrm{a}}$ \\
\hline $\mathrm{HDL}, \mathrm{mg} / \mathrm{dL}$ & $48.0(41.0-66.0)$ & $42.5(37.0-48.0)$ & $49.0(42.0-57.0)$ & $0.060^{b}$ \\
\hline $\mathrm{HDL}, \%$ & $29.2(19.0-33)$ & $21.1(18.0-29.6)$ & $26.0(22.0-32.0)$ & $0.191^{b}$ \\
\hline non-HDL, mg/dL & $143.5 \pm 39.8$ & $149.6 \pm 39.7$ & $149.1 \pm 43.9$ & $0.773^{\mathrm{a}}$ \\
\hline TG/HDL ratio & $2.3(1.4-4.0)$ & $3.7(2.4-5.4)$ & $2.9(2.0-5.6)$ & $0.090^{b}$ \\
\hline $\mathrm{LDL}, \mathrm{mg} / \mathrm{dL}$ & $121.5 \pm 33.9$ & $117.6 \pm 35.1$ & $108.0 \pm 36.6$ & $0.484^{\mathrm{a}}$ \\
\hline $\mathrm{TG}, \mathrm{mg} / \mathrm{dL}$ & $97.0(79.0-177.0)$ & $143.0(103.0-204.0)$ & $142.0(104.0-196.0)$ & $0.082^{b}$ \\
\hline
\end{tabular}

Normally distributed data are represented by mean \pm SD and skewed data by the median (interquartile range). $P<0.05$ is considered significant

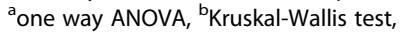

Abbreviations: HDL high-density lipoprotein cholesterol, HOMA-IR homeostatic model assessment of insulin resistance, LDL low-density lipoprotein cholesterol, TC total cholesterol, TG triglycerides, SI conversion factors: to convert TC, HDL, and LDL to $\mathrm{mmol} / \mathrm{L}$, multiply by 0.02586 ; TG to $\mathrm{mmol} / \mathrm{L}$, by 0.0114 ; glucose to $\mathrm{mmol} / \mathrm{L}$, by 0.05551 


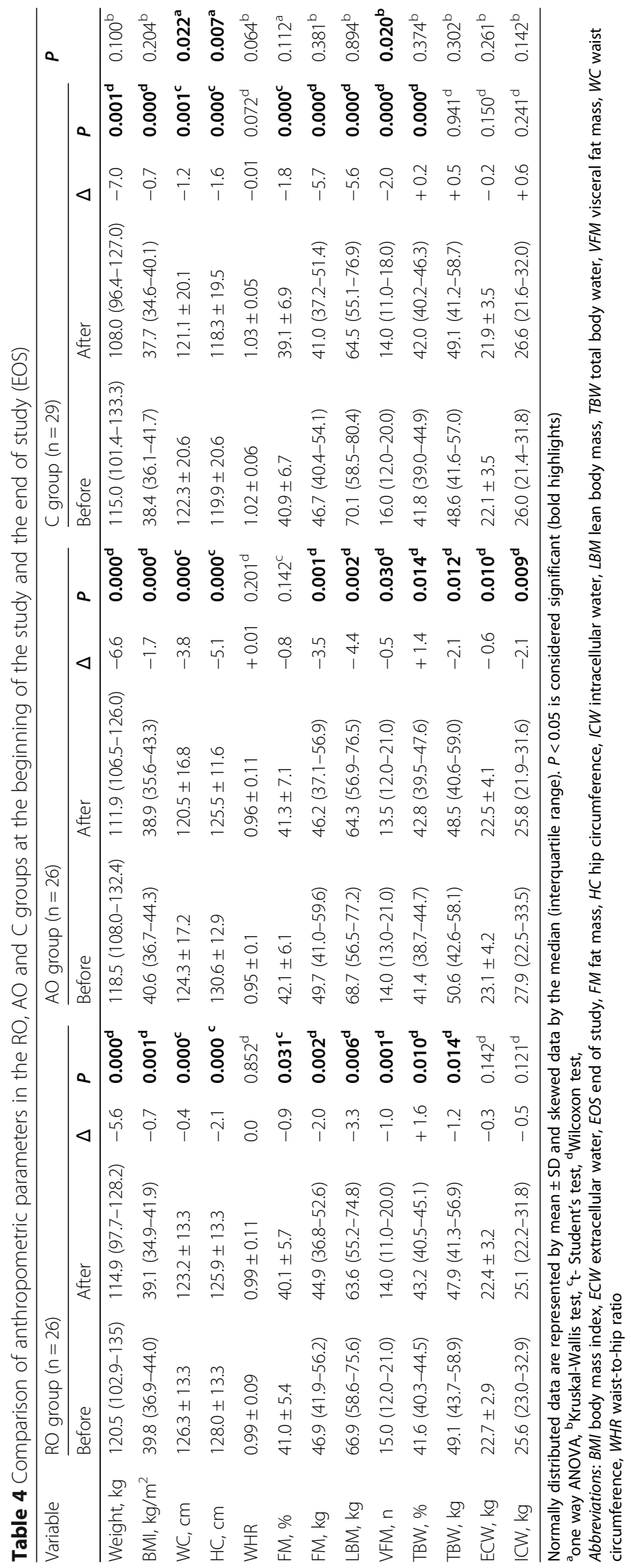




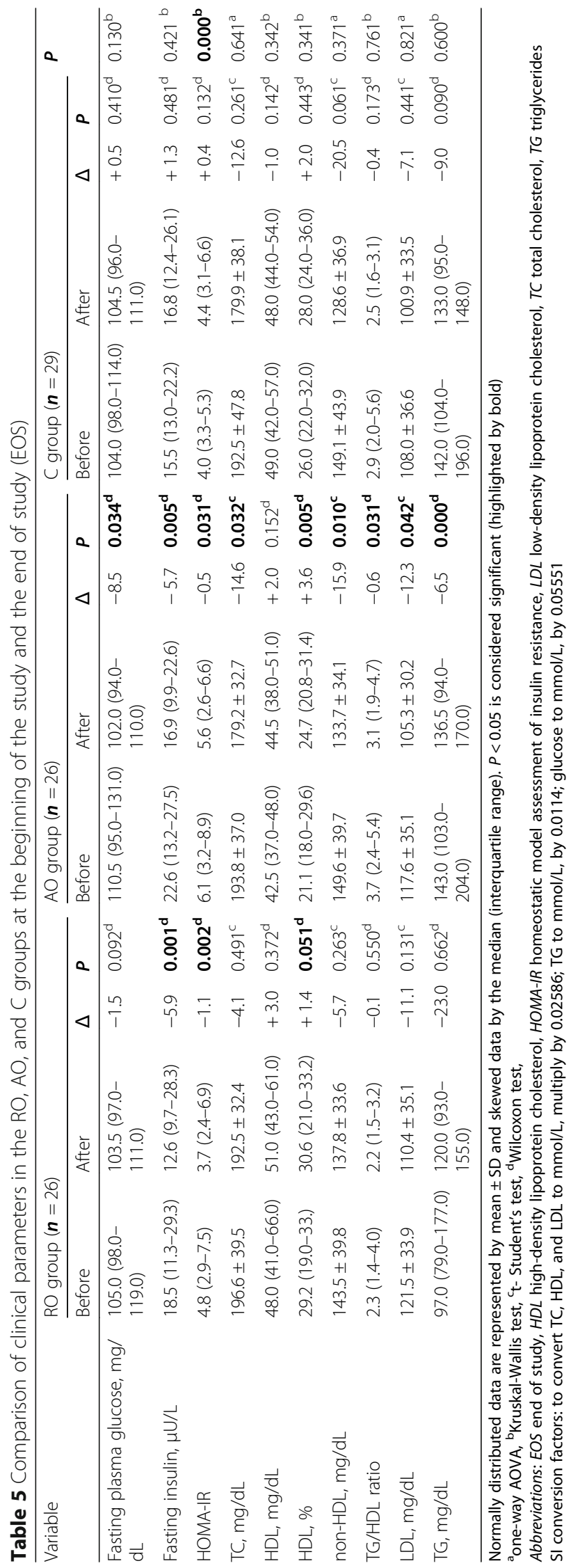


study were comparable with those observed in other studies $[29,30]$. It is noteworthy that the most significant reduction in VFM was observed in patients without oil supplementation, although it did not result in an improvement in metabolic parameters.

Numerous studies have indicated that consumption of high levels of MUFAs and PUFAs may improve glucose metabolism and lipid profile, compared to the consumption of fats containing higher levels of SFAs. However, whether replacement of dietary SFAs with higher concentrations of MUFAs or PUFAs would further enhance metabolic parameters remains unclear [31, 32]. A metaanalysis conducted by Qian et al. [33] revealed that, consumption of MUFA-rich diets resulted in significant reduction in fasting plasma glucose and a nonsignificant reduction in fasting insulin, TG, and LDL levels, compared to consumption of high-PUFA diets. By contrast, Miller et al. [32] demonstrated that substituting SFA with PUFAs in metabolic syndrome patients resulted in a higher reductions of TG and improved endothelial function than MUFAs.

The current study did not indicate any differences between intervention induced changes in the clinical parameters of $A O, R O$ and $C$, except in HOMA-IR. HOMA-IR was most markedly reduced in the OR group, while an increase in HOMA-IR was noticed in the C group. However, there was a trend toward significantly reduced fasting serum insulin levels and HDL\% in AO and $\mathrm{RO}$ groups, as opposed to the $\mathrm{C}$ group. Additionally, statistically significant changes in the fasting glucose level, TC, non-HDL, TG/HDL ratio, LDL and TG were observed in the AO group.

Previous studies have suggested that RSO may be used to normalize glucose profiles in humans [34-36]. A study of type 2 diabetes patients treated with an oral antihyperglycemic agent at a Canadian academic center, showed that consuming a canola oil-enriched lowglucose diet for 3 months improved glycemic control [34]. The effect of ASO on glucose metabolism was less clear. Kim et al., showed that 3 weeks of ASO supplementation $(100 \mathrm{mg} / \mathrm{kg})$ significantly reduced serum glucose levels in streptozocin-induced diabetic rats [11]. The beneficial effect of ASO in patients with diabetes mellitus type 2 has also been confirmed by Miroshnichenko et al. [37]. The current study observed significant improvements in fasting insulin levels and insulin sensitivity in the $\mathrm{AO}$ and $\mathrm{RO}$ groups, although changes in glucose levels were observed only in subjects supplemented with ASO.

The effect of RSO on circulatory cholesterol levels has been reported in most short-term interventions [38]. Lin et al. [7] demonstrated that diets rich in RSO resulted in substantial reductions in TC (12.2-12.5\%) and LDL levels (17\%). However, changes induced in HDL and TG levels by canola oil were evidently inconsistent. Furthermore, previous studies have reported that compared to consumption of high-SFA diets, consumption of diet enriched with RSO resulted in $8-10 \%$ reduction in HDL concentrations [39-41]. Data from this study showed that a calorie-restricted RSO-supplemented diet does not significantly affect TC and TG levels. A slight increase in HDL concentration and improvement in nonHDL and TG/HDL ratios were observed, but these changes were not statistically significant.

The beneficial effect exerted by ASO on cholesterol and bile acid absorption, cholesterol lipoprotein distribution, hepatic cholesterol content and cholesterol biosynthesis was demonstrated by Berger et al. [42], via an animal model study. In this study, hamsters were given hypercholesterolemic diets consisting of control, 10, or $20 \%$ Amaranthus cruentus grain, or 2.5 or $5 \%$ crude amaranth oil for 4 weeks. The results showed that amaranth oil (5\%) significantly decreased TC HLD and VLDL, compared to the control, and increased fecal excretion of particular neutral sterols and the bile acid, ursodeoxycholate [42]. However, an animal study [43] and a human pilot study [44] conducted by Berbger et al., revealed that cholesterol-lowering properties of ASO did not affect lipid profiles in an identical manner, and that the final effect of ASO on cholesterol metabolism may depend on factors such as amaranth species and cultivars, growing and processing conditions, as well as unique nutritional compositions.

Gonor et al. [45], investigated the beneficial effects exerted by a diet supplemented with squalene $(600 \mathrm{~mL} /$ d) from amaranth oil $(18 \mathrm{~mL} / \mathrm{d})$ on TC and TG concentrations and the fatty acid composition of erythrocytes, in patients with ischemic heart disease and hyperlipoproteinemia. Similarly, Martirosyan et al. [46], showed that 3 weeks of exposure to low-sodium/low-fat diets containing $\operatorname{ASO}(3,6,12$, or $18 \mathrm{~mL} / \mathrm{d})$ promoted positive dose-dependent changes in serum TC, LDL and TG levels in obese patients with coronary heart disease and hypertension.

The current study revealed that a 3-week intervention with ASO supplementation $(20 \mathrm{~mL} / \mathrm{d})$ led to a significant reduction in TC, \%HDL, LDL, and TG levels and caused a slight, nonsignificant, increase in HDL levels. Statistically significant improvements in non-HDL and TG/ $\mathrm{HDL}$ levels were also observed in the AO group. Although, ASO contains lower amounts of MUFA and LC n-3 PUFA than RSO, the presented study demonstrated that ASO caused more marked changes in lipid profiles than RSO. A better understanding of reasons underlying this finding may require further investigation.

The results of the current study showed that supplementation with ASO and RSO during the 3-week body mass reduction program did not cause changes in 
anthropometric measurements and clinical outcomes that were more effective, compared to group C. However, the study revealed a trend toward a more marked improvement in carbohydrate and lipid profiles in $\mathrm{AO}$ and $\mathrm{RO}$ groups compared with that in group $\mathrm{C}$.

\section{Study strengths and limitations}

The strength of this study is that the intervention was conducted under strictly controlled conditions. During the 3-weeks program, the participants were in the hospital ward, being under constant control over diet, supplementation and physical activity. The major limitations of this trial were small sample size and the relatively short duration of intervention. The main reason for such a short duration was that continued hospitalization for 3 weeks may interrupt professional and personal activities. However, it is noteworthy that presented study was one of the few studies conducted under specific and strictly controlled conditions, which is rare in nutritional interventions. Participants of the study received the same type of hypocaloric diet prepared by a dietetic food caterer and underwent the same physical activity program with a physical therapist. The 3-week hospitalization allowed the involvement of the study population in the intervention to be controlled. Although dual energy X-ray absorptiometry (DXA) is the gold standard for the assessment of body composition, the bioimpedance method (BIA) was used in the study due to its non-invasiveness, lower cost, and widespread use.

\section{Conclusions}

The results of this study indicated that although the 3week diet and physical activity program exerted a significant effect on anthropometric and clinical parameters, this effect was independent of ASO or RSO supplementation. However, ASO or RSO supplementation resulted in a trend toward more marked changes in the carbohydrate and lipid profiles of AO and RO groups, compared with those of the $C$ group. The use of therapies involving edible oils with high nutritional value, such as RSO and ASO, during body mass reduction programs, may enhance metabolic measurements.

\footnotetext{
Abbreviations

AO: Amaranth seed oil group; ASO: Amaranth seed oil; BIA: Bioimpedance; BMl: Body mass index; C: Control group; DXA: Dual X-ray absorptiometry; ECW: Extracellular water; FM: Fat mass; HC: Hip circumference; HDL: Highdensity lipoprotein; HOMA-IR: Homeostatic model assessment of IR; ICW: Intracellular water; LBM: Lean body mass; LC n-3 PUFAs: Long chain polyunsaturated fatty acids; LDL: Low-density lipoprotein;

MUFA: Monounsaturated fatty acids; RO: Rapeseed oil group; PAL: Physical activity level index; PUFA: Polyunsaturated fatty acids; RSO: Rapeseed oil; SFA: Saturated fatty acids; TBW: Total body water; TC: Total cholesterol; TDEE: Total daily energy expenditure; TG: Triglycerides; USFA: Unsaturated fatty acids; VFM: Visceral fat mass; WC: Waist circumference; WHO: World Health Organization; WHR: Waist-to-hip ratio
}

\section{Acknowledgments}

Not applicable.

\section{Trial registration}

DRKS, DRKS00017708. Registered 05 September 2019 - Retrospectively registered, https://www.drks.de/drks_web/navigate.do?navigationld=trial. HTML\&TRIAL_ID=DRKS00017708

\section{Authors' contributions}

$M M, A Z$, and $M G$ conceived the concept of the study. MM, AZ, and MG contributed to the design of the research. MM, $A Z$, and $A J$ were involved in data collection and statistical analysis. MM, AZ, and PB contributed to data interpretation and drafting of the article. MG and PB coordinated the project tasks. All authors edited, revised, and approved the final version of the manuscript.

\section{Funding}

Not applicable.

\section{Availability of data and materials}

The datasets used and analyzed during the current study are available from the corresponding author on reasonable request.

\section{Ethics approval and consent to participate}

Consent was obtained from each participant included in the study. The study was approved by the Bioethics Committee at Poznan University of Medical Sciences (No. 333/14) and was performed in accordance with the Declaration of Helsinki.

\section{Consent for publication}

Not applicable.

\section{Competing interests}

The authors declare that they have no competing interests.

\section{Author details}

${ }^{1}$ Department of Obesity and Metabolic Disorders Treatment and Clinical Dietetics, Karol Marcinkowski University of Medical Sciences, ul. Szamarzewskiego 82/84, 60-569 Poznań, Polska, Poland. ²Department of Gastroenterology, Dietetics and Internal Medicine, Karol Marcinkowski University of Medical Sciences, Poznan, Poland. ${ }^{3}$ Centre of Pulmonology and Thoracic Surgery, Poznan, Poland.

Received: 26 January 2020 Accepted: 17 June 2020

Published online: 20 June 2020

\section{References}

1. Baboota RKB, Bishnoi M, Ambalam P, Kondepudi KK, Sarma SM, Boparai RK, et al. Functional food ingredients for the management of obesity and associated comorbidities - a review. J Funct Foods. 2013;5:997-1012.

2. Obesity and overweight. Key facts. World Health Organization. 2020. https:// www.who.int/en/news-room/fact-sheets/detail/obesity-and-overweight. Accessed 10 Nov 2019.

3. Rodriguez JE, Campbell KM. Past, present and future of pharmacologic therapy in obesity. Prim Care. 2016;43:61-7.

4. Cicero AFG, Fogacci F, Colletti A. Food and plant bioactives for reducing cardiometabolic disease risk: an evidence based approach. Food Funct. 2017:8:2076-88.

5. Martirosyan DM, Singh J. A new definition of functional food by FFC: what makes a new definition unique? Funct Food Health Dis. 2015;5:209-23.

6. Grajeta H. Functional foods in prevention of cardiovascular disease. Adv Clin Exp Med. 2004;13:503-10.

7. Lin L, Allemekinders H, Dansby A, Campbell L, Durance-Todd S, Berger A, et al. Evidence of health benefits of canola oil. Nutr Rev. 2013;71:370-85.

8. Nasirpour-Tabrizi P, Azadmard-Damirchi S, Hesari J, Piravi-Vanak Z. Amaranth Seed Oil Composition. Nutritional Value of Amaranth. 2020. https://www. intechopen.com/books/nutritional-value-of-amaranth/amaranth-seed-oilcomposition. Accessed 20 Feb 2020.

9. Boczar P, Błażejczyk-Majka L. Characteristic of vegetable oil consumers in Poland in a view of sustainable consumption principles. Acta Sci Pol Oeconomia. 2015;14:15-26. 
10. Moszak M, Zawada A, Grzymisławski M. The properties and the use of rapeseed oil and amaranth oil in the treatment of metabolic disorders related to obesity. Forum Zaburzeń Metabolicznych. 2018;9:53-64.

11. Kim HK, Kim MJ, Cho HY, Kim E-K, Shin DH. Antioxidative and anti-diabetic effects of amaranth (Amaranthus esculantus) in streptozotocin-induced diabetic rats. Cell Biochem Funct. 2006;24:195-9.

12. Maniak B, Zdybel B, Bogdanowicz M. Ocena wybranych właściwości fizykochemicznych tradycyjnych olejów roślinnych produkowanych na ziemi lubelskiej. Agric Eng. 2012;3:101-7.

13. Kyle UG, Bosaeus I, De Lorenzo AD, Deurenerg P, Elia M, Gomez JM, et al. Bioelectrical impedance analysis-part II: utilization in clinical practice. Clin Nutr. 2014;23:1430-53.

14. Yang R, Xue L, Zhang L, Wang X, Qi X, Jian J, et al. Phytosterol contents of edible oils and their contributions to estimated Phytosterol intake in the Chinese diet. Foods. 2019:8:334.

15. Venskutonis PR, Kraujalis P. Nutritional components of Amaranth seeds and vegetables: a review on composition, properties, and uses. Comp Rev Food Sci Food Safety. 2013;12:381-412.

16. Munro Hazard B. Statistical methods for health care research. 5th ed. Lippincot Williams \& Wilkins; 2005.

17. Knopfholz J, Disserol CC, Pierin AJ, Schirr FL, Streisky L, Takito LL, et al. Validation of the Friedewald formula in patients with metabolic syndrome. Cholesterol. 2014;2014:261878.

18. Katsuki A, Sumida Y, Gabazza EC, Murashima S, Furuta M, Araki-Sasaki R, et al. Homeostasis model assessment is a reliable indicator of insulin resistance during follow-up of patients with type 2 diabetes. Diabetes Care. 2001:24:362-5.

19. Marusteri M, Bacarea V. Comparing groups for statistical differences: how to choose the right statistical test? Biochemia Med. 2010;20:15-32.

20. Perez-Cornago A, Brennan L, Ibero-Baraibar I, Hermsdorff HHM, O'Gormann A, Zulet MA, et al. Metabolomics identifies changes in fatty acid and amino acid profiles in serum of overweight older adults following a weight loss intervention. J Physiol Biochem. 2014;70:593-602.

21. Kim SK, Karadeniz F. Biological importance and applications of squalene and squalane. Adv Food Nutr Res. 2012;65:223-33.

22. Hammad SS, Jones PJ. Dietary fatty acid composition modulates obesity and interacts with obesity-related genes. Lipids. 2017:52:803-22.

23. Norris LE, Collene AL, Asp ML, Hsu JC, Liu L-F, Richardson JR, et al. Comparison of dietary conjugated linoleic acid with safflower oil on body composition in obese postmenopausal women with type 2 diabetes mellitus. Am J Clin Nutr. 2009;90:468-76.

24. Borsonelo EC, Vieira L, Galduróz JC. The influence of the polyunsaturated fatty acids on body weight and anxiolytic-like behavior in female rats. Nutr Neurosci. 2013;16:2-5.

25. Munro IA, Garg ML. Prior supplementation with long chain omega-3 polyunsaturated fatty acids promotes weight loss in obese adults: a doubleblinded randomized controlled trial. Food Funct. 2013;4:650-8.

26. Riserus $U$, Berglund L, Vessby B. Conjugated linoleic acid (CLA) reduced abdominal adipose tissue in obese middle-aged men with signs of the metabolic syndrome: a randomized controlled trail. Int J Obes Metab Disord. 2001:25:1129-35.

27. Kaippert VC, Santos Lopes MC, Carvalho PD, Rosaldo DE. Effects of unsaturated fatty acids on weight loss, body composition and obesity related biomarkers. Diabetol Metab Syndr. 2015;7:A139.

28. Krishnan S, Cooper JA. Effect of dietary fatty acid composition on substrate utilization and body weight maintenance in humans. Eur J Nutr. 2014;53: $691-710$.

29. González-Muniesa P, Mártinez-González MA, Hu FB, Després JB, Matsuzawa Y, RJF L, et al. Obesity. Nat Rev Dis Prim. 2017;3:17034.

30. Sartorio A, Agosti F, Resnik M, Lafortuna CL. Effects of a 3-week integrated body weight reduction program on leptin levels and body composition in severe obese subjects. J Endocrinol Investig. 2003;26:250-6.

31. Rathod KJ, Jadeja BA, Jadav JK, Golakiya BA. Physico-chemical characterization and fatty acid profiling of seed oils of grain amaranthus cultivars of India-a nutritional perspective. Int J Pure App Biosci. 2017:5: 1424-32.

32. Miller M, Sorkin JD, Mastella L, Sutherland A, Rhyne J, Donnelly P, et al. Poly is more effective than monounsaturated fat for dietary management in the metabolic syndrome: the muffin study. J Clin Lipidol. 2016;10:996-1003.

33. Qian F, Korat AA, Malik V, Hu FB. Metabolic effects of monounsaturated fatty acid-enriched diets compared with carbohydrate or polyunsaturated fatty acid-enriched diets in patients with type 2 diabetes: a systematic review and meta-analysis of randomized controlled trials. Diab Care. 2016;39:144857.

34. Jenkins DJ, Kendall CW, Vuksan V, Faulkner D, Augustin SA, Mitchell S, et al. Effect of lowering the glycemic load with canola oil on glycemic control and cardiovascular risk factors: a randomized controlled trial. Diabetes Care. 2014;37:1806-14.

35. Uusitupa M, Schwab U, Mäkimattila S, Karhapää P, Sarkkinen E, Maliranta $H$, et al. Effects of two high-fat diets with different fatty acid compositions on glucose and lipid metabolism in healthy young women. Am J Clin Nutr. 1994:59:1310-6.

36. Södergren E, Gustafsson IB, Basu S, Nourooz-Zadeh J, Nälsén C, Turpeinen A, et al. A diet containing rapeseed oil-based fats does not increase lipid peroxidation in humans when compared to a diet rich in saturated fatty acids. Eur J Clin Nutr. 2001;55:922-31.

37. Miroshnichenko LA, Zoloedov VI, Volynkina AP, Kulakova SN. Influence with amaranth and sunflower oils used in dietary therapy of patients with diabetes mellitus 2 types on parameters of carbohydrate and lipid metabolism. VoprPitan. 2008;77:53-7.

38. Gillingham LG, Gustafson JA, Han SY, Jassal DS, Jones PJH. High-oleic rapeseed (canola) and flaxseed oils modulate serum lipids and inflammatory biomarkers in hypercholesterolaemic subjects. Br J Nutr. 2011;105:417-27.

39. Gustafsson IB, Vessby B, Ohrvall M, Nydahl M. A diet rich in monounsaturated rapeseed oil reduces the lipoprotein cholesterol concentration and increases the relative content of n-3 fatty acids in serum in hyperlipidemic subjects. Am J Clin Nutr. 1994;59:667-74.

40. Lichtenstein AH, Ausman LM, Carrasco W, Jenner JL, Gualtieri B, Goldin R, et al. Effects of canola, corn, and olive oils on fasting and postprandial plasma lipoproteins in humans as part of a national cholesterol education program step 2 diet. Arterioscler Thromb. 1993;13:1533-42.

41. McDonald B, Gerrard J, Bruce V, Corner EJ. Comparison of the effect of canola oil and sunflower oil on plasma lipids and lipoproteins and on in vivo thromboxane $\mathrm{A} 2$ and prostacyclin production in healthy young men. Am J Clin Nutr. 1989;50:1382-8.

42. Berger A, Gremaud G, Baumgartner M, et al. Cholesterol-lowering properties of amaranth grain and oil in hamsters. Int J Vitam Nutr Res. 2003;73:39-47.

43. Berger A, Monnard I, Dionisi F, Gumy D, Hayes KC, Lambelet P. Cholesterollowering properties of amaranth flakes, crude and refined oils in hamsters. Food Chem. 2003;81:119-24.

44. Berger A, Monnard I, Bilat M. Effects of highly palatable amaranthcontaining food on cholesterol levels of hypercholesterolemic men: pilot study. Proc Germ Nutr Soc. 2000;2:28.

45. Gonor KV, Pogozheva AV, Kulakova SN, Medvedev FA, Miroshnichenko LA. The influence of diet with including amaranth oil on lipid metabolism in patients with ischemic heart disease and hyperlipoproteidemia. Vopr Pitan. 2006;75:17-21.

46. Martirosyan DM, Miroshnichenko LA, Kulakova SN, Pogojeva AV, Zoloedov V. Amaranth oil application for coronary heart disease and hypertension. Lipids Health Dis. 2007;6:1.

\section{Publisher's Note}

Springer Nature remains neutral with regard to jurisdictional claims in published maps and institutional affiliations.

Ready to submit your research? Choose BMC and benefit from:

- fast, convenient online submission

- thorough peer review by experienced researchers in your field

- rapid publication on acceptance

- support for research data, including large and complex data types

- gold Open Access which fosters wider collaboration and increased citations

- maximum visibility for your research: over $100 \mathrm{M}$ website views per year

At $\mathrm{BMC}$, research is always in progress.

Learn more biomedcentral.com/submissions 